\section{A Case Report of COVID-19 Positive Becker Muscular Dystrophy}

\author{
Lakshmi Prasanna Digala ${ }^{1} \mathrm{MD}$; \\ Shivika Prasanna ${ }^{3} \mathrm{MS}$; Praveen $\mathrm{Rao}^{2,3} \mathrm{PhD}$; \\ Adnan Qureshi ${ }^{1,4} \mathrm{MD}$; Raghav Govindarajan ${ }^{1} \mathrm{MD}$. \\ ${ }^{1}$ Department of Neurology, University of Missouri \\ Health Care, Columbia, MO, USA 65201. \\ 2 Department of Health Management \& Informatics, \\ University of Missouri. Columbia, MO, USA 65201. \\ ${ }^{3}$ Department of Electrical Engineering \& Computer \\ Science, Center for Biomedical Informatics, \\ University of Missouri. Columbia, MO, USA 65201. \\ ${ }^{4}$ Zeenat Qureshi Stroke Institute. St. Cloud, \\ Minnesota 56301.
}

\section{ABSTRACT}

Background: Becker and Duchenne muscular dystrophies constitute the most common inherited dystrophinopathies. The chronic steroid treatment predisposes them to any infection, hence, we sought to determine the current COVID-19 infection in them. We conducted an analysis on a real-world database to identify the effect of COVID-19 infection and identified a case of Becker muscular dystrophy who tested positive for COVID-19. For our analysis, we utilized Cerner Real-World Data ${ }^{\mathrm{TM}}$ that was provided through Cerner's HealtheDataLab research tool.

Case report: A 63-year-old Caucasian male with Becker muscular dystrophy, hyperlipidemia, and atrial fibrillation, was hospitalized with COVID-19 infection. Our search revealed June 22, 2020, as the patient's COVID-19 service date when tested positive. The patient received antibiotics and supportive therapy during hospitalization. Intricate details like oxygen requirement, blood gas analysis, and mechanical ventilation could not be retrieved if used. The patient developed complications like sepsis, pneumonia, acute respiratory failure that resulted in prolonged hospitalization. Our data reported that the patient was alive during discharge.

Conclusion: Although patient developed complications during hospitalization, no death from the COVID-19 infection was observed in our analysis.

Keywords: Becker Muscular Dystrophy, COVID-19 infection, Muscular dystrophy.

\section{Background}

Becker and Duchenne muscular dystrophies constitute the most common inherited dystrophinopathies. Becker muscular dystrophy (BMD) is an X-linked recessive disorder characterized by progressive muscle weakness, secondary to the truncated dystrophin gene's low levels. This gene encodes for a protein called dystrophin-4, and the incidence is one-third as frequent as Duchenne muscular dystrophy (DMD), which is a severe form. ${ }^{1,5}$ They present with progressive muscle weakness, and the chronic steroid treatment predisposes them to any infection., ${ }^{1,2}$ The current global pandemic COVID-19 infection presents with a dry cough, fever, headache, shortness of breath, and reduced sensation of smell. In severe cases, it results in pneumonia, acute respiratory failure, myocardial injury, and death. ${ }^{2}$ COVID-19 infection has several implications on the course of various neuromuscular diseases. We conducted an analysis on a real-world database to identify the effect of COVID-19 infection on BMD/DMD patients.

\section{Case Report}

For our analysis, we utilized Cerner Real-World Data ${ }^{\mathrm{TM}}$ that was provided through Cerner's HealtheDataLab research tool. ${ }^{4}$ The COVID-19 dataset in HealtheDataLab contains de-identified patient data of one hundred and seventeen thousand patients from 62 contributing health systems. The dataset contained all patients tested for COVID-19 at some point during their visits to one of the 62 health centers. All patients with the DMD/BMD tested for COVID-19 were identified using ICD-10-CM code (G71.01) and SNOMED-CT code (76670001). This yielded a total of 5 patients that were tested either positive or negative for COVID-19. Out of them, two patients were aged 17 (DMD), and the other three (BMD) ranged from 38-63. One of the five patients tested positive for COVID-19 infection on June 22, 2020 and was hospitalized. Our Institutional Review Board waived the consent form as we utilized deidentified patient data.

The patient identified was a 63 -year-old Caucasian male, and comorbidities identified were hyperlipidemia and atrial fibrillation. The duration of hospitalization in the patient was 28 days. The patient received antibiotics and supportive therapy during hospitalization. Intricate details like oxygen requirement, blood gas analysis, and mechanical ventilation could not be retrieved if used. The complications identified during the hospital stay were pneumonia, atrial fibrillation, sepsis, and respiratory failure. The data reported that the patient was alive during discharge; however, the discharge disposition could not be retrieved as it was listed as an empty value in the database. 


\section{Discussion}

NotethatBMD patients are pronetodevelop COVID-19 infection and complications. The immunocompromised state in these BMD patients secondary to steroid treatments predisposes them to infections and complications. ${ }^{1,2,3}$ As per a consensus recommendation, there are no significant changes in the standard of care for BMD patients. ${ }^{2,3}$ They also reported providing appropriate care must be targeted to prevent the adrenal crisis in these patients when they fall ill. Higher doses of corticosteroids are recommended in those scenarios. ${ }^{2}$

The common cause of death in BMD patients is cardiac dysfunction/cardiomyopathy. ${ }^{5}$ In our case the patient developed sepsis and acute respiratory failure from the COVID-19, which resulted in prolonged hospitalization of almost a month.

Although the patient developed complications during hospitalization, no death from the COVID-19 infection was observed in our analysis.

\section{References}

1. Flanigan KM. Duchenne and Becker muscular dystrophies. Neurologic clinics. 2014 Aug 1;32(3):671-88.

2. Veerapandiyan A, Wagner KR, Apkon S, et al. The care of patients with Duchenne, Becker, and other muscular dystrophies in the COVID-19 pandemic. Muscle Nerve. 2020 Apr 24.

3. Stratton AT, RO RI, Kupfer O, Carry T, Parsons J, Apkon S. Pediatric neuromuscular disorders: Care considerations during the COVID-19 pandemic. J. Pediatr. Rehabil. Med. 2020 Oct 30 .

4. Ehwerhemuepha L, Gasperino G, Bischoff N, et al. HealtheDataLab-a cloud computing solution for data science and advanced analytics in healthcare with application to predicting multi-center pediatric readmissions. BMC Med Inform Decis Mak. 2020 Jun 19;20(1):115.

5. Verhaert D, Richards K, Rafael-Fortney JA, Raman SV. Cardiac involvement in patients with muscular dystrophies: magnetic resonance imaging phenotype and genotypic considerations. Circ Cardiovasc Imaging. 2011 Jan;4(1):67-76. 